\title{
The Value Exploration of EAP's Psychological Counseling Work in Independent Colleges - Take Hetianmu Center of the School of Management as an Example
}

\author{
Tianwen Yang ${ }^{1, \mathrm{a}}$ and Guangli Luo ${ }^{2, \mathrm{~b}, *}$
}

\begin{abstract}
${ }^{I}$ School of business administration, guangdong university of foreign studies south china business college, guangzhou, guangdong, China

${ }^{2}$ School of business administration, guangdong university of foreign studies, guangzhou, guangdong, China

a2035612930@qq.com, ${ }^{b} 2362404297 @ q q . c o m$

*Corresponding author
\end{abstract}

\begin{abstract}
Nowadays, EAP has been introduced into China, and it is widely used in enterprises with remarkable effects. But the use of EAP is by no means limited to this, nor should it be limited to this. Today's college students are a bundle of pressures. In independent colleges, the combination of EAP and peer counseling can effectively reduce the occurrence of psychological crisis and extreme events among college students. The combination of EAP, peer counseling and independent colleges will be the general trend, and its advantages of low cost, wide coverage and high utility make it have unlimited potential. This paper discusses the value of EAP's psychological counseling work in independent colleges, and hopes to provide useful data for psychological counseling work in independent colleges and our school.
\end{abstract}

Keywords: psychological crisis EAP, Hetian center, peer counseling, stress

\section{EAP 在独立学院心理辅导工作的价值探索一一以管院二 级禾田姆中心为例}

\author{
杨添文 ${ }^{1, a}$, 罗光丽 ${ }^{1, b, *}$
}

1 管理学院, 广东外语外贸大学南国商学院, 广州, 广东, 中国

a2035612930@qq.com, ${ }^{b} 2362404297 @ q q . c o m$

*通讯作者

\section{摘要}

EAP 已传入我国, 多在企业中运用且效果显著。其实 EAP 之用途绝不仅限于此应。研究表明: EAP 的功能很广, EAP 必将对组织发展起着不可替代的作用。当今大学生集众多压力源于一身。因此在独 立院校中，通过与朋辈咨询的结合可以有效地降低大学生心理危机以及极端事件的发生。EAP、朋辈 咨询、独立院校三者的结合将是大势所趋，其成本低、覆盖广、效用高的优点使之潜力无限。本文就 此探讨 EAP 在独立学院心理辅导工作的价值, 并希望能给各个独立院校以及我校的心理咨询工作提 供有用的数据。

关键词：心理危机 EAP，不田姆中心，朋辈咨询，压力

\section{1. 序}

\section{1. 背景}

把高考政策改革写进去更好, 是政策导致大学生普及, 加上社会的需求两方面原因。随着大学门槛的逐渐降低,
大学生数量急剧增加, 大学生心理疾病问题日益突出, 尤其在面对学习、生活、感情及未来社会工作压力等多 重因素下, 大学生心理问题逐渐成为各大高校较为重要 的问题。【1】大学生的情绪自我的调节能力变差, 心理 的抗压能力降低, 很容易就走极端。就在上半年, 很多 院校都发生了多起跳楼自杀案件。这其中 有研究认为, 相比于同龄非大学生群体, 大学生心理疾病问题较为突 出, 从比重来看, 近 2-3 成的大学生患有不同程度的心 理疾病, 1 成左右的大学生患有较为严重的心理疾病。 
我们设计了包含基本情况、心理状况、心理干预三大方 面的调查问卷，通过以禾田姆中心的名义在网上发布给 同学们, 以此来了解同学们的心理状况。问卷具体内容 见附录。我们应用客观的态度和科学的方法, 对大学生 中常见现象, 在本院校范围内进行实地考察, 并搜集大 量资料, 结合抽样调查法和统计调查法进行数据的收集 与发现。并通过随机发放 50 份匿名问卷调查我们发现了 有百分之八十三的人都有明显 的感受到压力，如下表：

\section{2. 意义}

随着大学生心理疾病问题的日益突出, 大学生心理健康 状况逐渐成为全社会所关心的问题。【3】如今大学仅有 的心理咨询机构和心理咨询老师远不能满足几千甚至上 万学生的心理咨询需求, 若增加常设咨询师就会增加经 费负担和造成人力资源的浪费。而引进 EAP 就能很好地 解决此问题。首先 EAP 作为一个专门应对心理危机的救 援团体, 它的专业性冊庸置疑, 其次它可以以最少的成 本给学生提供更全面的心理援助。这将对独立院校的学 生心理健康、心理素质、人身安全、学校秩序管理极其 有利。EAP 原本有助于员工安心, 提高学生的忠诚度。 若是用于独立院校, 则对学生起着巨大的作用, 它可以 有效的减少学生中不可控因素、提升学生自我管理能力、 提高学生学习效率等。【4】让学生视校如家。由于目前 学校无法更多增加咨询力量的时候, 朋辈咨询保持作用 价值的特殊性、朋辈之间的认同性、关系建立容易。

\section{3. 目的}

本次探究的目的在意, 对外希望使大家充分了解心理咨 询、明白 EAP 价值所在, 能够引起大家对 EAP 进校园 的充分重视。此外更希望这些探索能够给于各个独立学 院对于建立校园 EAP 作为一个数据的参考。对内希望能 够引起校方对 EAP 进校园的重视, 以及激发相关部门对 大学生心理健康、EAP 与大学生的创新。望本院校能够 支持并推广 $\mathrm{EAP}$ 与校园心理健康的结合, 以及对管院二 级禾田姆中心的推广。使每个院都能建立这样的中心, 使 EAP 惠及每个学生。让目前现有的咨询条件的情况下 通过朋辈咨询使有身心成长需求的学生得到及时帮助、 咨询服务供需严重失衡的状况得到改善。

\section{4. 方法}

为了 EAP 在独立学院心理辅导工作的价值探索中获得更 多有用的数据, 我们用了包括问卷调查法 与网络调查法 相结合、实地调查法、抽样调查法、统计调查法, 等多 种调查方法。 


\section{心里是否有感受到明显压力}

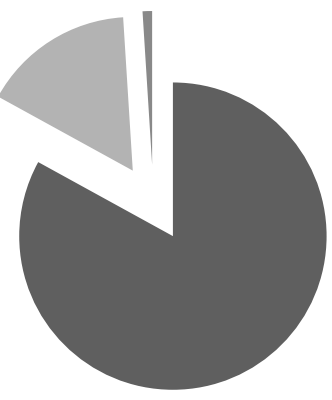

有

没有

口不知道

图 1 心理压力调查统计表

而在百分之八十的人之中, 感到有过心情低落, 什么事

情都不想做的, 则高达百分之八十九:

\section{是否因压力而感到心情低落什么 事都不想做}

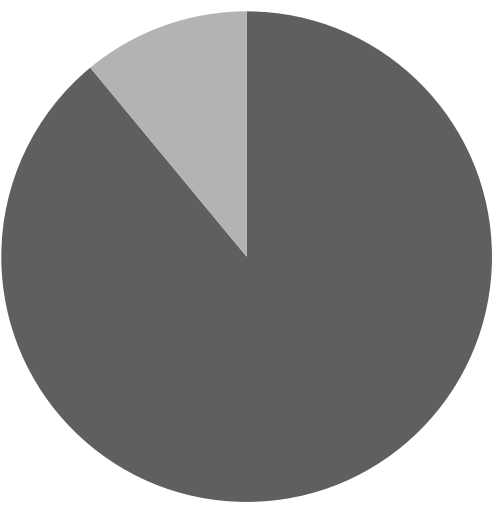

是

图 2 压力影响调查统计表

当中是否有找人倾诉的比例是找人倾诉百分之四十三, 而不找的百分之五十五, 还有纠结的百分之二, 而这百 分之四十三种, 觉得有效果的只有百分之十八: 


\section{是否找人倾诉}

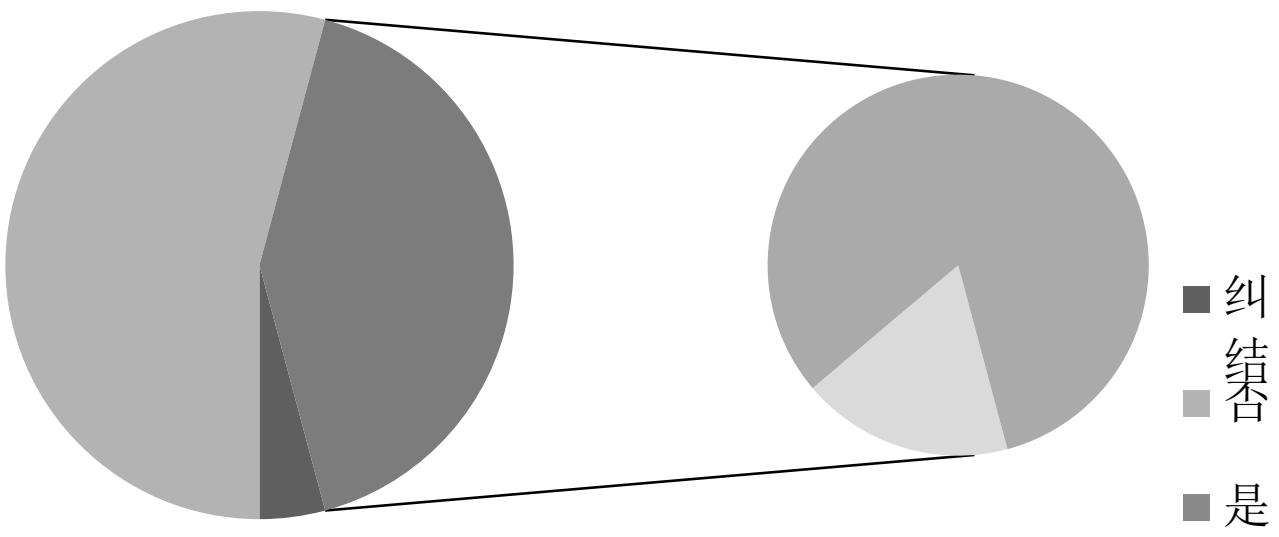

图 3 是否找人倾述调查统计表

在问道为什么不招人的倾述的时候, 最多的回答便是因

为因为陌生人件的保密性不高。

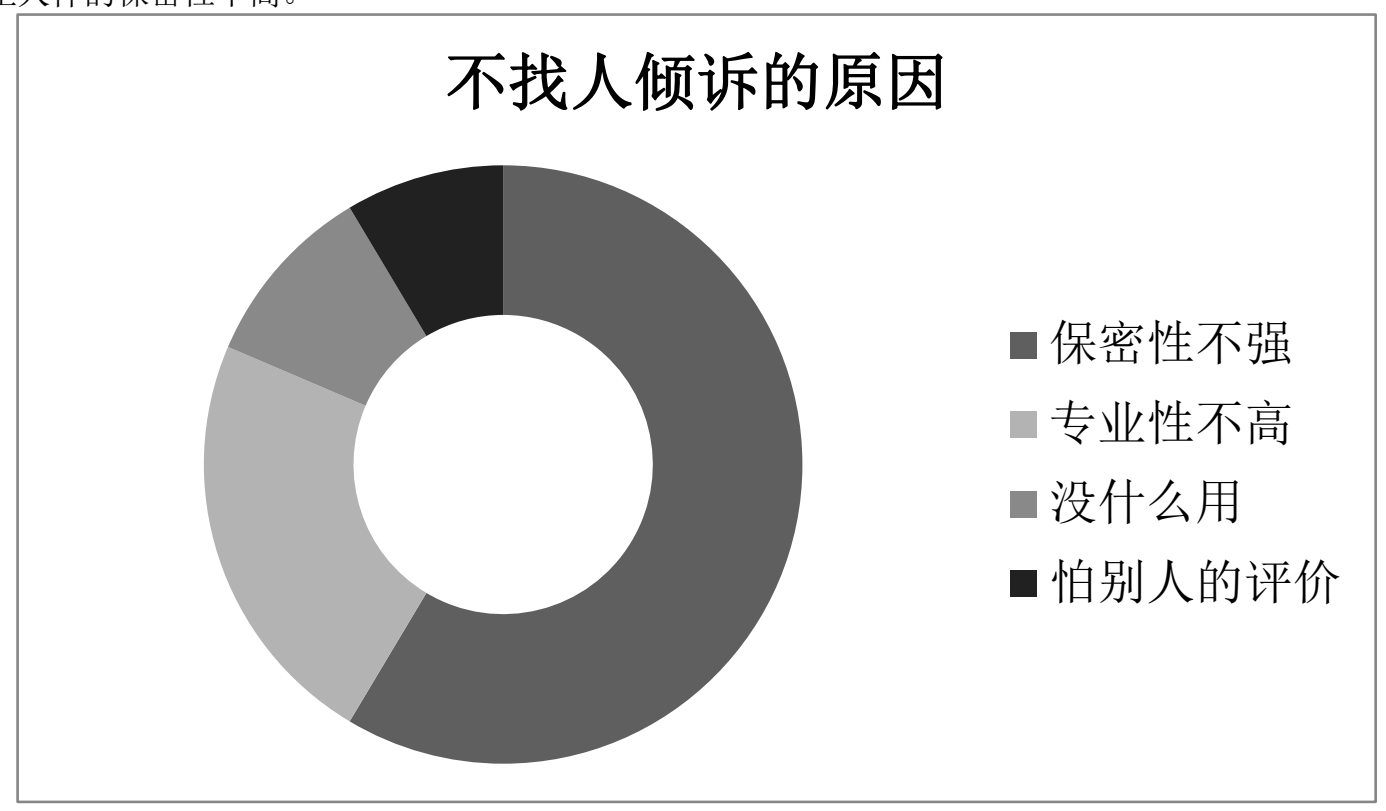

图 4 不找人倾诉原因调查统计表

这些结果反应了, 在院校中心理咨询的需求量大但信任 感不足的问题。

\section{2. 方案}

\section{1. 做了什么}

EAP 在国外来说已经不是一个陌生的名词了, 但在国内 它才刚起步。虽然如此, 但它在国内却呈现星星之火的
态势, 短时间内影响力扩散的速度有目共睹。在企业中 更是如此。那么 EAP 到底是什么? 它究竟有什么价值呢? 首先 EAP(Employee Assistance Program)的含义为员工帮 助计划, 可帮助企业对自己的员工予以科学的、系统的、 稳定的心理健康引导;经过专业人士进行评估, 为员工健 康状况保驾护航, 可有效提升员工心理问题的抵御能力, 进一步提升企业员工凝聚力。【5】人心理和行为健康 (在国外通常的说法是精神健康, 即 Mental Health) 是 EAP 最为关心的层面, 通过系统化的追踪及引导, 保证 员工处于健康的心理状态。【6】其次, 随着我国大学生 心理健康问题重视程度不断加强, EAP 管理理念及心理 健康输送方式逐渐得到了较大的认可。尤其近几年来我 国加强了对外交流，国外先进的 EAP 管理理念逐渐引入 
传播大学心理健康知识为宗旨, 通过系列的心理活动等

到国内诸多企业及高校, 逐渐在对学生或员工的心理指 导起到关键性作用。与此同时, 随着互联网信息化的不 断发展, EAP 管理理念逐渐得到了国内诸多企业级高校 的重视, 尤其国内大学生心理疾病现象日益严重的情况 下, EAP 方法的运用是后续高校管理学生心理健康问题 最为主要的方式。

那它到底有什么样的价值我们用一组数据来说话。在 $\mathrm{EAP}$ 投入方面, 投入 1 美元, 可为企业降低 5 美元左右 的运营成本。EAP 在企业的运用逐渐受到重视, 为企业 员工提供长期的、科学的心理辅导与疏通, 不但可以显 著提升员工凝聚力, 提高员工工作效率, 还能够使员工 保持健康的心理状态。【7】据统计, $85 \%$ 以上的世界 500 强企业引入了 EAP 管理模式, 并将之作为管理员工 心理健康的主要模式和方法。麦当劳总结以往经验发现, 任职 90 天内离开公司的员工, 会给公司直接或间接带来 555 美元以上的损失。1987 年, 麦当劳公司报告了在他 们的 EAP 计划上被花费的每一元都有 4: 1 的回报。最 近, 公司报告投资上的回报高达 10: 1。康菲石油公司 引入 EAP 后发现, 企业员工能举例及工作效率得到了较 为明显的改善, 员工心里疾病患有率得到显著下降, 整 体员工工作氛围较为健康。【8】

EAP 员工鼓励项目能够为企业员工提供长期的、稳定的、 科学的心理监管, 为企业员工心理健康提供良好的保障。 而独立学院作为私立学校, 不属于事业单位是以企业的 形式而存在的, 所以企业说面临的问题独立学院依然也 会面临。所以独立学院需要 EAP, 而 EAP 在独立学院更 能体现价值。因为其价值体现不仅在员工而且更多的是 在学生。EAP 被引入学生群里是历史的必然, 如今沈阳 民办二本高校已经开始探索 EAP 与民办高校相结合的模 式。他们通过培训使大部分的人得到了观念的更新和转 变; 掌握了一些自我与协调的技巧; 对压力管理和咨询 管理也有了一些自己的认识。【9】但是沈阳二本民校的 引入了只是很浅层次的引入且覆盖范围不够广多扣人数 不够多。这说明 EAP 进校园仍然有较大的发展空间, 仍 然具有发展的潜力。为什么这样说, 因为如今虽然有 EAP 进校园这个概念但, 培训和咨询的人员大多是以心 理老师为主。这就出现了全校这么多人师生比例悬殊难 以一对一、点对点的进行咨询和跟进。这就需要朋辈咨 询的引入了。

朋辈咨询是近几年发展较为迅速的心理疏通模式, 其主 要特点集中在参与心理辅导的人员与被辅导人员年龄相 仿、处境相似, 通过同类人群的引导治疗, 能够迅速融 入心理疾病患者的心理状态, 朋辈咨询因为所处环境类 似、年龄阶段相仿, 因而能够迅速获得患者的注意, 进 而能够实现较好的心理辅导效果。【10】

朋辈心理辅导与传统专业的心理治疗模式存在一定的差 异性, 由于其具有同类性, 因此其往往能够实现更好的 心理治疗效果。一般意义上来说, 朋辈咨询专业性不高, 但如果和 EAP 相结合呢? 把 EAP 融入到朋辈咨询中去, 组建朋辈咨询团队。这样医治成本低而且效用大覆盖面 广的 EAP 队伍就产生了。其实这不是泛泛而谈, 管院二 级禾田姆中心就是这么一支队伍。我们在心理咨询需求 日益增长, 且急需创新的情况下产生。我们以帮助独立 院校的学生身心灵健康发展为目标。虽然正在探索阶段。 但已经取得了不错的成果。禾田母新家园, 这是一个二 级心理救助站。禾田姆心家园隶属管理学院, 是一个以
方式能够起到帮助大学生解决心理困惑、心理压力的作 用, 同时传播心理健康知识, 帮助同学们认识自我、发 展自我、改变自我。禾田姆心家园名字解释：自性的禾 苗在肥沃的土壤里汲取养分, 健康茁壮成长。 


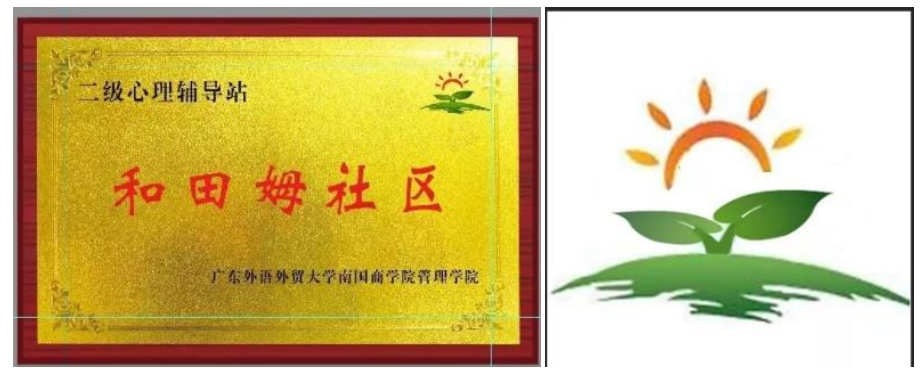

图 5 禾田姆心灵家园牌匾和标志

虽然禾田姆成立的时间不长, 还在摸索着 EAP 与大学生 身心健康结合的道路之中，但朋辈咨询的人数已有五人， 而五人中解决问题, 能够改变心理状况的人数也有五人, 成功率百分之一百。此外我们还成功的举办了一场绘画 治疗和一场心理知识培训。在培训上同学们学习到了, “心理基础知识”, “心理异常表现”, “大学生心理 健康认识与心理发展路程”, “精神断奶与大学生生命 健康” “大学生心理危机识别与预警”、“大学生的生 命困难” , “如何做一个有效的救助者” 等很多作为朋 辈咨询的知识。自利才能利他, 通过培训, 我们不仅收 获到如何帮助身边的人和发现身边人的心理危机状况。 更多的是更能读懂自己, 调节自己提高自己的抗压能力 和自我情绪管理能力。禾田姆工作人员做过随机调查反 馈, 发现陪训前和培训后感觉到更能认识到自我和得到 自我心理问题的解决的比例为 $75 \%$ 。还有绘画治疗, 在 绘画治疗中, 我们主要的工作是通过收集同学们的作品, 让专业老师看出同学们的性格和心理状况。一次有针对 性的进行心理辅导。这是有助于大范围排除大学生心理 危机的一个很好的方法。绘画治疗, 对不不善表达不敢 表达着无疑是一个表达内心述求的突破口。

大学, 作为一个特殊的阶段, 一方面它是另一种学习方 式的开始阶段, 另一方面它也是全职学习模式的准结束 阶段。【11】所以无论是大一到大四我们都有各自的压 力都有可能产生心理危机。大一新生, 他们面临的是自 律与他律、能力还是成绩的问题。独立学院作为自主学 习的殿堂, 与高中老师严加看管, 老师天天推着学习, 除学习外什么事都有老师父母包办不同, 独立学院是一 个自主性很高的地方, 在这自己要为自己负责, 不单是 学业还有生活人际关系等, 在这没有人为你包办, 什么 事都得靠自己; 在这里师傅领进门修行靠自己; 在这人 与人的流动性很高, 你和你的同学不是经常带在一起, 你的老师也不是固定的位置等你找他。在这种突然间被 迫长大急需成长的环境下就如加了增长剂的小苗被拉伸
的很辛苦。这个时候肯定会出现压力会出现负面情绪。 这就很需要朋辈的心理辅导心理引导。因为你经历的朋 辈也在经历所以他的话就很有感染力和说服力了。这样 就可以很好地避免和解决大一新生入学的心理危机了。 到了大二随着专业的深入, 为求到对方的认同也为了专 业的需要, 就开始各类的考级和考证了, 学习方面的压 力就会加重了很多。随之而来的英语四、六级, 计算机 等级考试, 各项专业技能等级考试以及考研热、读博热 还有出国热等等, 这些大小不一的考试让他们又回到了 为考试而奔波的时代, 种种压力让大家背上了一个沉重 的包袱。

到了大三, 随着在校时间的减少, 学业压力加重, 如果 这时四级以及专业证书未能拿到那么自我的负面情绪加 深, 心理危机就会出现, 这也是为什么大三是极端事件 频发的一个阶段。向后看碌碌无为向前看毕业论为, 以 及生存就业压力步步逼近。这时焦虑压力混合一起产生 巨大的心理负担。再加上家人的期望, 更加加深大学生 的心理危机状况。经调查结果显示在平时学习生活中, 有 $62 \%$ 的学生觉得父母对自己的期望高而给自己带来压 力, 有 $20 \%$ 的人觉得压力很大, 觉得没有压力的仅占 $4 \%$ 。这个时候这些压力就会十分的凸显。这个时候如果 是心理咨询老师未必能融入他们的心理状况, 只能是朋 辈才能感同身受畅所欲言, 在畅所欲言的过程中对他们 进行心理上的疏导, 解除他们的心理危机。到了大四就 业、毕业论文、专业是否与工作对口、爱情是否能继续。 这些就需要融入了 EAP 的朋辈咨询团队来进行心理咨询 了。因为这需要的更多是帮助其进行职业生涯规划。

所以, 朋辈咨询以及 EAP 对于大学的每一个阶段都发挥 着不可或缺的作用。每个大学阶段不同的心理危机的应 对更需要 EAP 给予在理论层面上的支撑。以及以及进一 步的探索。经过我们团队一学期的能力, 我们设置了禾 田姆服务模式, 具体模型如下: 


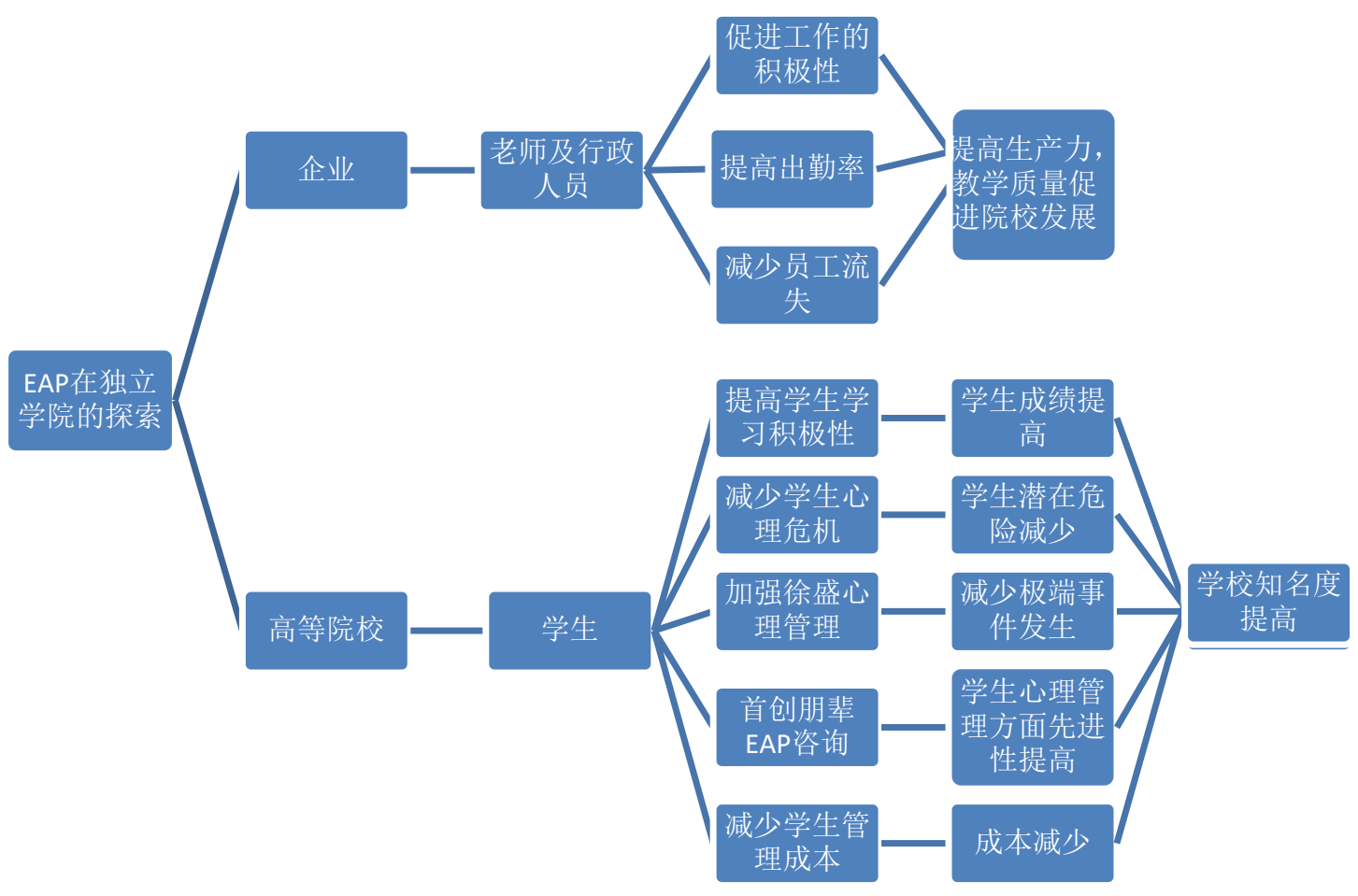

图 $6 \mathrm{EAP}$ 在独立学院的探索规划图

\section{3. 结论}

\section{1. 规划}

我校管院二级禾田姆中心后期的规划与发展分为三个阶 段，第一阶段前期通过讲座活动来发现问题经历与校方 沟通等去解决问题后期影响力足够时发动同学们去发现 导致大学生心理健康问题的因素, 并且通过搜集到的信 息设法去为建立一个积极的, 支持性的, 和健康的学习 环境而努力第二阶段培训志愿者。通过给他们培训分级, 例如: 针对不同年级的学生产生的不同问题, 产生心理
危机不同因素, 是怎样对学生心理健康产生影响, 以及 相应的提高对抗不良心理问题的能力的方法。有关的教 育课程包括应付工作压力, 自信性训练, 放松技术, 生 活问题指导, 以及解决问题技能等。当他们身边的朋友 出现相关的问题的时候, 他们便可用上这些知识去对他 们进行简单的心理疏导。同时平时也可以帮助身边的朋 友提高心理抗压能力。以及定时的开展针对不同年级的 心理咨询的讲座培训, 让同学们不仅可以通过他力解决 问题也可以通过自身的能力从源头上排除负面情绪。第 三完善朋辈心理咨询团队的建设, 形成一个完整的运作 体系。这种心理咨询服务如果有可能的话后期通常也面 向同学的直系家庭成员。整个流程如下: 


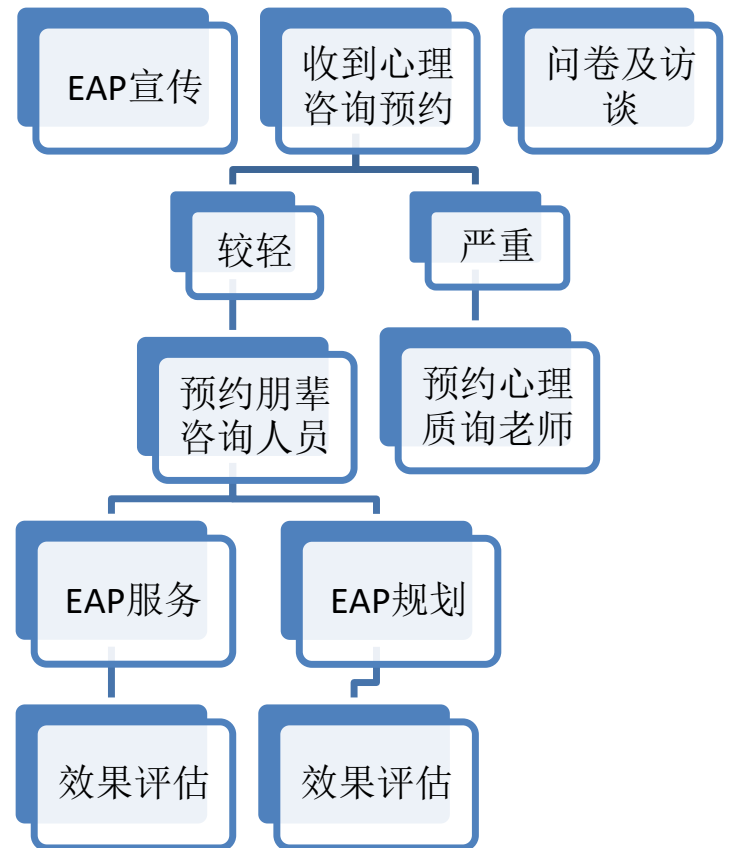

图 7 禾田姆心灵家园工作流程图

我国国各类高等教育在学总规模达到 3699 万人, 高等教 育毛入学率达到 $42.7 \%$ 。【12】全国共有普通高等学校 和成人高等学校 2880 所, 高职 (专科) 院校 1359 所, 全国共有研究生培养机构 793 个。这么庞大的人群, 每 个人的压力加起来之巨大可想而知。每所学校每个学院 建立像禾田姆这样的援助站的需要迫切程度之高而想而 知。所以开展 EAP 在独立学院心理辅导工作的价值探索 是必不可少的工作。希望这篇文章能给予各个独立院校 的开展 EAP 在独立院校的工作有一个数据的参考。

\section{2. 结论}

引入 EAP 进入独立院校与朋辈咨询相结合是当前独立学 院 EAP 工作模式实施的现实需要, 它有助于解决学生日 益增多的心理危机与独立学院心理咨询老师不足, 心理 咨询力量较为薄弱之间矛盾的困境; 促进学生之间相互 沟通, 和情绪自我管理能力的提升; 更有效的减少犀利 咨询成本的同时获得覆盖面较广的学生心理状况管理, 因此, 朋辈 EAP 模式在独立院校的应用中是支强有力的 生力军, 可以活动于校园的的各个角落。

而禾田姆中心是这种模式的一个开端。而且它的实践也 证明了, 在大学中开展 EAP, 建立相应的心理援助站, 组建朋辈咨询团队是有必要的。因为我们的建立, 从正 式成立那一刻起我们才经历了三个月不到的时间, 但我 们的效果, 影响, 成绩斐然。地上本无路, 只是走的人 多了就自然成了路。希望各独立院校也可以与我们开展 EAP 在独立学院心理辅导工作的价值探索, 尽我们之力 以最低的成本降低大学生的心理危机, 让大学生与独立 院校一起健康发展。
[1] 宋宝萍. 《大学生心理健康教育》 [M]. 西安电子科技 大学出版社. 2007 年 8 月, 36-78.

[2] 段金金星, 程婧. 《大学生心理危机干预》 [M]. 科学出版 社. 2006 年 1 月, 43-98.

[3] 何金彩, 唐闻捷. 《大学生心理健康与发展》 [M]. 浙江 大学出版社. 2005 年 8 月, 56-79.

[4] 潘海红. 《大学生心理健康自助》 [M]. 合肥工业大学 出版社. 2006 年 8 月 $10-, 36$.

[5] 赵然. 《员工帮助计划——中国经典案例集》 $[\mathrm{M}]$. 科 学出版社. 2017 年 3 月 28-,65.

[6] 张西超. 《员工帮助计划》 [M]. 中国人民大学出版社. 2015 年 8 月, 26-55.

[7]《心理学与生活》 [M]. (美) 里查理・格里格、菲利 普・津巴多著王垒译. 人民邮电出版社. 2003 年 10, 1-16. [8] 《影响力》 $[\mathrm{M}]$. (美) 西奥迪尼 著, 问佳 译. 万卷出 版公司. 2010 年 9 月, 33-97.

[9]《微反应心理学》 $[\mathrm{M}]$. 陈璐 编著. 中央编译出版社. 2013 年 10 月, 21-63.

[10] 《行为心理学》 [M]. 龙春华 著. 中国华侨出版社. 2012 年 08 月, 67-98.

[11]《当代教育心理学》 [M]. 陈琦, 刘儒德 主编. 北京师 范大学出版社. 2007 年 04 月, 25-46.

[12] 快资讯:

[Z]http://www.360kuai.com/pc/9a88686f2436a5664?cota=3 \&kuai_so=1\&tj_url=wd\&sign=360_57c3bbd1\&refer_scene $=$ so_1

\section{参考文献}


附录

\begin{tabular}{|c|}
\hline 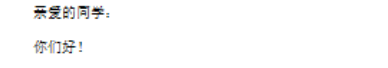 \\
\hline 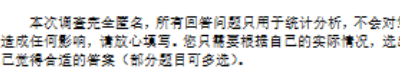 \\
\hline 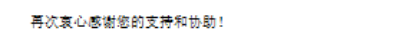 \\
\hline 一、基本情况 \\
\hline 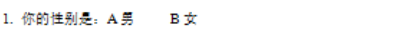 \\
\hline 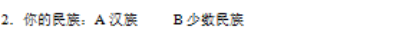 \\
\hline 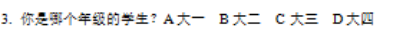 \\
\hline 4. 你的专业军列? \\
\hline 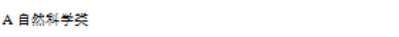 \\
\hline 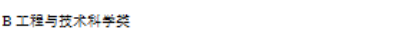 \\
\hline c人文与社会絃军基 \\
\hline 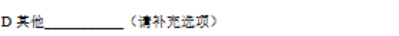 \\
\hline 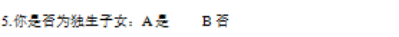 \\
\hline 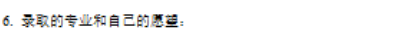 \\
\hline A 非茛待合、清尊 \\
\hline B 比教法尊 \\
\hline$c-f_{2}$ \\
\hline D不太清首 \\
\hline 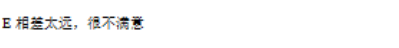 \\
\hline
\end{tabular}

7. 你是否遇到心理问题?

$\mathrm{A}$ 经常有 $\mathrm{B}$ 偶尔有 $\mathrm{C}$ 没有过 $\mathrm{D}$ 没有想过

8. 目前你最大的苦恼是什么? (可多选)

A 学习压力大

B 就业压力大

C 家庭经济压力大

D 人际关系紧张

E其他——请补充选项)

9. 你是否接受过心理咨洵? A 是 B 否

$$
\text { 二、心理状况 }
$$

1.你认为人际交往 :

A 很重要 B 无所谓

2.你对自己的人际交往感到:

A 轻松梌快

B 与个别人难以交往

C 与他人交往平淡、缺少知心朋友

D紧张困难

3.你认为自己的人际关系:

A 较好 B - 般 C较差

4.你有几个知心朋友: ——

5. 如果你不愿意进行人际交往, 原因是:

$\mathrm{A}$ 与他人难以沟通

B 不相信他人 
5. 你认为一个心理健康的人最典型特征是什么?

A 处事乐观, 热情诚恳

B 心平气和, 与世无争, 乐于助人

C 有良好的人际关系

6. 你认为造成大学生轻生的原因有哪些? (可多选)

A 学习压力大

$B$ 对自己的专业不满意

C 家庭条件比别人差

$\mathrm{D}$ 失恋

$\mathrm{E}$ 毕业后找不到工作

F人际关系失败

$\mathrm{H}$ 其他

7. 你认为轻生的大学生存在以下哪种问题? (多选)

$\mathrm{A}$ 缺亏社会责任感

B 不能真正理解 “死究竟意味着什么”

C 心理受挫能力差

$\mathrm{D}$ 适应力能差

$\mathrm{E}$ 缺少对人生值价观的认识

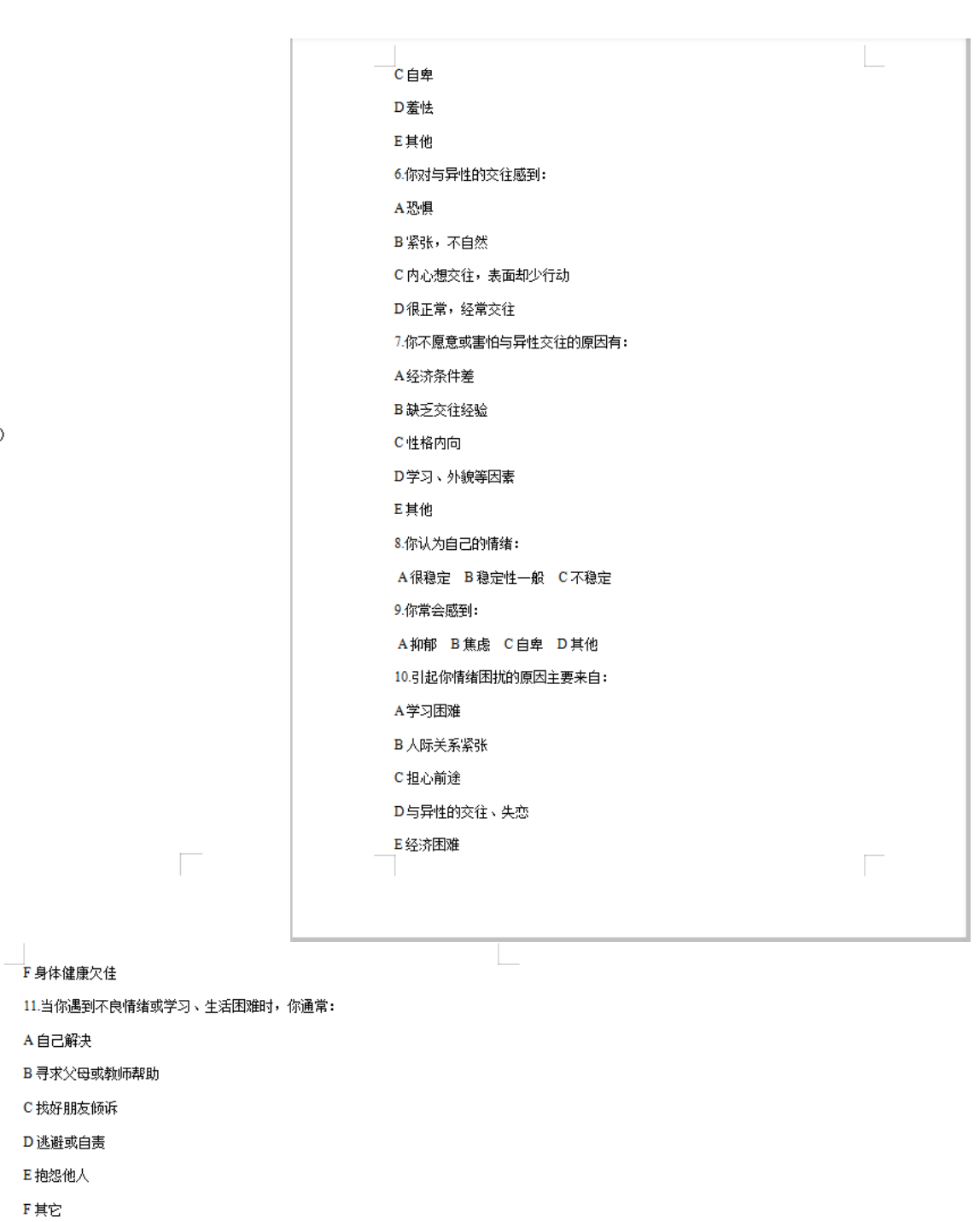

$$
\text { 三、心理干预 }
$$

1.学校是否有心理健康咨洵室?

$\mathrm{A}$ 有 $\mathrm{B}$ 没有

2.如果你身边的朋友存在心理问题, 你会如何做?

A 开导他

B 事不关己, 无所谓

C 对外宣传他有问题

3.你认为大学生的心理健康是否应引起重视?

A 要认真对待 B 无所谓 C 根本不用在意

4. 你认为学校目前最应该加强哪方面的心理教育工作? (可多选)

A 进行心理健康知识宣传

B 举行心理健康讲座与染询

C 进行心理健康状兄调查

$D$ 深入同学中多与同学沟通

E网络心理咨询 